\title{
3D skin model to investigate the early epidermal morphological psoriatic features
}

\author{
Donetti E ${ }^{*}$, Lombardo G', Baruffaldi Preis $F^{2}$, Cornaghi L ${ }^{1}$, Pescitelli $\mathrm{L}^{3}$ and Prignano $\mathrm{F}^{3}$ \\ ${ }^{1}$ Department of Biomedical Sciences for Health, Università degli Studi di Milano, 20133 Milan, Italy \\ ${ }^{2}$ Plastic Surgery Unit, Ospedale Niguarda Ca' Granda, Milano \\ ${ }^{3}$ Department of Surgery and Translational Medicine, Università degli Studi di Firenze, 50125 Florence, Italy
}

\begin{abstract}
Epidermal cells, together with different molecules of the immunosystem, promote psoriasis, but several data concerning the early phases of this disease still lack. This study evaluated the early morphological features of psoriasis using an organotypic culture of normal human skin. Cellular proliferation, expressions of Toll-like receptors (TLR) 7 and 9, and keratin 17 were analysed by indirect immunofluorescence in normal human skin biopsies exposed to a cytokine mix strictly reproducing a psoriatic environment. After mix incubation, an early and progressive decrease of cell proliferation was detected. TLR9 was present in the granular layer of mix samples, while TLR7 was expressed throughout the entire epidermal compartment. K17 expression was evident after mix exposure. In conclusion, our results prove that a psoriatic microenvironment is able to induce pivotal morphological changes in our skin model, strongly suggesting that an early epidermal "psoriatic switch" can be reproduced for studying the epidermal homeostasis and innate immune response.
\end{abstract}

\section{Introduction}

Psoriasis is a common chronic inflammatory skin disease with a significant socio-economic impact and negatively affecting patients' quality of life. An interplay of genetic factors and many external stimuli as skin injuries, environmental influences, and microbial infections [1] can trigger or worsen the disease. This disorder is characterized by an intricate pathogenic mechanism involving both immune system and cell types [2]. Keratinocytes are key cells in innate immunity due to both their peculiar ability to recruit $\mathrm{T}$ cells to the skin and their unique role in epidermal skin barrier. After triggering signals, keratinocytes are able to produce many antimicrobial peptides as cathelecidin LL37, many defensins, and S100 proteins [3,4]. They do also behave as secretive cells, because they can produce high levels of chemochine CCL20 able in further recruiting T-cells into the skin [5]. Finally, yet importantly, keratinocytes express Toll Like Receptors (TLRs) which allow to specifically respond against environmental microbial challenges [6]. There are many clinical and experimental evidences concerning the importance of TLR-mediated inflammation in psoriasis and the development of therapeutics that negatively modulate TLRs signaling pathways; the related effects in psoriasis pathogenesis are highly required [7].

Up to now, most of data are obtained on late phases of psoriasis, when skin lesions are already consolidated, epidermal psoriatic plaques are mostly hyperkeratotic, and dermis infiltrated by different cell types. For this reason, for better understanding the early phases of the pathogenesis of this disease, models that mimic the disease as close as possible and that can be used to develop new therapies are mandatory. To help in defining the very early phase of the disease, in the last decade we used a simple but well standardized three-dimensional organotypic culture model of normal human skin biopsies after exposure to pivotal pro-inflammatory psoriatic cytokines [8-11] in different associations.
Thanks to this firm background, we can now use our 3D model to investigate how all these cytokines together can influence the psoriatic phenotype and to determine their effects on keratinocytes. In particular, we focused our attention on the analysis on TLR expression, K17 induction, and keratinocyte proliferation in the epidermal compartment evoked by a psoriatic microenvironment represented by a mix of cytokines containing TNF-alfa, IL-17, IL-22 and IL-23.

\section{Methods}

\section{Organotypic cultures}

Bioptic fragments of normal human skin were obtained after aesthetic surgery from healthy young non-smoking women after written informed consent $(n=7)$. The procedures were in accordance with the ethical standards of the Institutional Committee on human experimentation and with the Helsinki Declaration. After surgery, samples were overnight incubated in a Transwell system (Costar, Corning, NY, USA) epidermal side-up at the air-liquid interface to reduce the acute mechanical stress as previously reported [8-11]. Samples were cultured in Dulbecco's modified Eagle's culture medium (Euroclone, Milan, Italy) containing 10\% fetal bovine serum (FBS; Invitrogen, Life Technologies Ltd, Paisley, UK) supplemented with penicillin/streptomycin, amphotericin B, and glutamine (SigmaAldrich, St. Louis, MO, USA). No hydrocortisone was added to avoid

${ }^{\star}$ Correspondence to: Elena Donetti, Department of Biomedical Sciences for Health, Universita degli Studi di Milano, Via Mangiagalli, 31 - 20133 Milan, Italy, E-mail: elena.donetti@unimi.it

Key words: adaptive immunity, toll-like receptors, keratin 17, keratinocyte proliferation, indirect immunofluorescence

Received: October 27, 2019; Accepted: November 14, 2019; Published: November 18, 2019 
an anti-inflammatory activity. After overnight incubation fragments were exposed to a mix of TNF-alpha $100 \mathrm{ng} / \mathrm{ml}$, IL-17 $50 \mathrm{ng} / \mathrm{ml}$, IL$22100 \mathrm{ng} / \mathrm{ml}$ and IL-23 $50 \mathrm{ng} / \mathrm{ml}$ for 24 (T24), 48 (T48) or 72 (T72) hours. All cytokines were from PeproTech (London, UK). Parallel samples were incubated in absence of a medium without any cytokine and represented our internal controls. Each patient was represented at all experimental points and in all groups. In order to evaluate epidermal cell proliferation, all bioptic samples were incubated for 3 $\mathrm{h}$ with 5-bromo-2'-deoxyuridine (BrdU), a non-radioactive thymidine analogue selectively incorporated in DNA of S-phase cells. Samples $(5 \times 5 \mathrm{~mm})$ were then formalin-fixed, paraffin-embedded, and cut by a microtome obtaining serial section of $5 \mu \mathrm{m}$ thickness.

\section{Immunofluorescence analysis}

In all experiments, paraffin sections were dewaxed, rehydrated in a descending scale of ethanols, treated for antigen unmasking and nonspecific binding site saturation before incubation with specific primary and secondary antibodies. Rabbit anti-human TLR7 and mouse antihuman TLR 9 antibodies were purchased from Novus Bio, while mouse anti-human K17 antibody from Cell Signaling Technology. The antigen unmasking for TLR7 and TLR9 was performed in autoclave in Tris $\mathrm{HCl}$ buffer $0.05 \mathrm{M} \mathrm{pH} 8.5$ for $20^{\prime}$ at $98^{\circ} \mathrm{C}$ or in citrate buffer $0.01 \mathrm{M} \mathrm{pH} 6$ for $9^{\prime}$ at $120^{\circ} \mathrm{C}$, respectively. After the saturation of unspecific sites with goat serum $1: 10$ in PBS $0.1 \mathrm{M}$ with BSA $2 \% / 30^{\prime}$ at RT, TLR7 antibody was diluted 1:300 in PBS 0.1M with 2\% BSA and TLR 9 was diluted 1:10 in PBS $0.1 \mathrm{M}$. Slides were then incubated at $4^{\circ} \mathrm{C}$ overnight. As secondary antibody we used for TLR7 a goat anti-rabbit (Alexa Fluor 488) 1:100 diluted in PBS $0.1 \mathrm{M}$ with BSA $2 \% / 1 \mathrm{~h} \mathrm{RT}$ and for TLR9 a goat anti-mouse FITC-conjugated (Jackson Immunoresearch) 1:200 in PBS 0,1M/ 1h RT. K17 immunofluorescence analysis was performed similarly to TLR9 protocol with a dilution of the primary antibody 1:500 in PBS/BSA 1\%. One section on each slide was considered as technical negative control and the primary antibody was thus omitted and replaced with $0.1 \mathrm{M}$ phosphate-buffered saline (PBS), $\mathrm{pH}$ 7.4. Nuclei were counterstained with 4, 6-diamidino-2-phenylindole dihydrochloride (DAPI 1:50000 dilution in bi-distilled water; 5 min of incubation at RT. All histological and immunofluorescence analysis were performed utilizing a Nikon Eclipse E600 equipped with a Nikon digital camera DXM1200 (Nikon, Tokyo, Japan).

\section{Quantitative analysis of epidermal proliferation}

BrdU incorporation was revealed using a monoclonal antibody anti-BrdU (Santa Cruz Biotechnology, Dallas, TX; 1:200, 60 min) after DNA denaturation with $2 \mathrm{~N} \mathrm{HCl}(30 \mathrm{~min})$, buffering with $\mathrm{Na}_{2} \mathrm{~B}_{4} \mathrm{O}_{7}(10$ $\mathrm{min})$, saturation of non-specific binding sites with $3 \%$ bovine serum albumin (Sigma-Aldrich, St. Louis, MO, USA) in PBS (20 min), and digestion with $0.05 \%$ pepsin in $20 \mathrm{mmol} \mathrm{L}^{-1} \mathrm{HCl}(25 \mathrm{~min})$. A FITCconjugated horse anti-mouse IgG (Vector Laboratories, Burlingame; 1:200, $60 \mathrm{~min}$ ) was used as secondary antibody. All procedures were performed at room temperature (RT) except for pepsin incubation, which was done at $37^{\circ} \mathrm{C}$. For the analysis of cell proliferation, at least three experiments were carried out for each sample, with two slides per sample and two sections on each slide. Each section was considered as a sample unit for statistical analysis. Two independent, double-blinded investigators counted BrdU-positive cells. The results were expressed as mean number + standard deviation (SD) of BrdUpositive cells per $\mathrm{mm}^{2}$ of epidermis containing nucleated cells. Epidermal area was calculated on adjacent haematoxylin and eosinstained sections, excluding the stratum corneum. The software Image Pro-Plus (version 4.5.019; Media Cybernetics, Silver Spring, MD) was used for the measurement [13].

\section{Results}

\section{TLR7 expression}

In samples exposed to the cytokine mix, starting from T24, TLR7 was homogeneously expressed throughout the entire epidermal compartment at all time points and TLR7-positive cells were present also in the basal layer (Figure 1A and 1B). Immunopositivity at T72 appeared weaker than at earlier time points (Figure 1C) and particularly localized in the lower less differentiated layers. In healthy skin (Control), as previuosly demonstrated [11], TLR7 was never expressed in the basal monolayer and its distribution slightly increased towards the epidermal surface in the more differentiated epidermal layers.

\section{TLR9 expression}

TLR9 immunopositvity started to be present at T24 only in the granular layer of mix samples (white arrows in Figure 1D). The immunostaining was more and more evident in the granular layer in mix samples both at T48 and T72 (white arrows Figure 1E and 1F). As expected, TLR9 was never expressed in control samples (data not shown).

\section{K17 expression}

K17 immunostaining was always negative in control samples (Figure 2A), in accordance to previous data from our and other labs [10]. At T24, after the incubation with the cytokine mix, a progressive increase of K17 expression was evident in the cytoplasm of keratinocytes in the lower layers of the epidermal compartment (Figure 2B). At T48, K17 immunoreactivity was evident mostly in the upper spinous layer (Figure 2C). At T72 the entire epidermal compartment was K17positive, excluding the basal layer (Figure 2D).
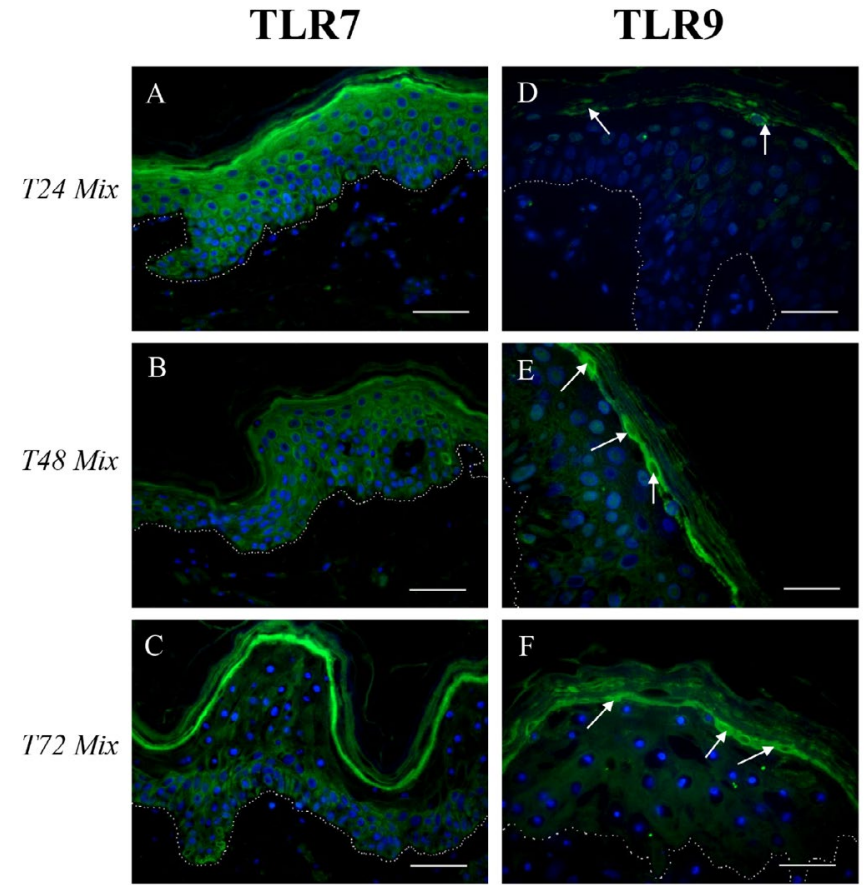

Figure 1. (A-C) TLR7 and (D-F) TLR 9 immunostaining on paraffin skin sections of samples incubated with medium containing TNF-alpha, IL-17, IL-22, and IL-23. (A, D): T24; (B, E): T48; (C, F): T72. TLR7: Toll-like receptor 7; TLR9: Toll-like receptor 9. T24 Samples harvested 24 hours after the overnight incubation; T48: Samples harvested 48 hours after the overnight incubation; T72: Samples harvested 72 hours after the overnight incubation. Dotted white line indicates the basal membrane; white arrows indicate TLR9 localization in the uppermost granular layer. Bars: $50 \mu \mathrm{m}$ 


\section{Quantitative analysis of epidermal proliferation}

We observed a progressive decrease of keratinocyte proliferation in control samples (Figure 3, white bars), accordingly to our previous data $^{8}$. The incubation with the cytokine mix induced a strong inhibition of cell proliferation starting 24 hours after the overnight incubation (Figure 3, black bars). A statistical significant difference was observed at T48 and T72 between control and mix samples $(\mathrm{p}<0.05)$.

\section{K17}

Control

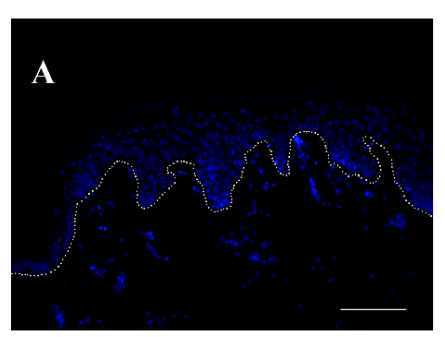

T48 Mix
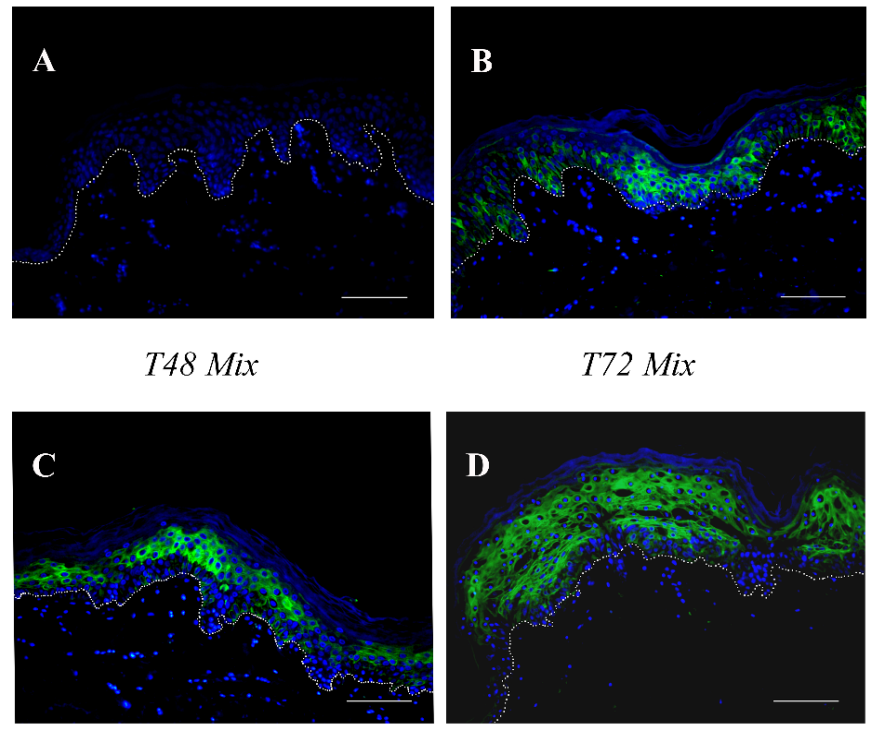

T72 Mix

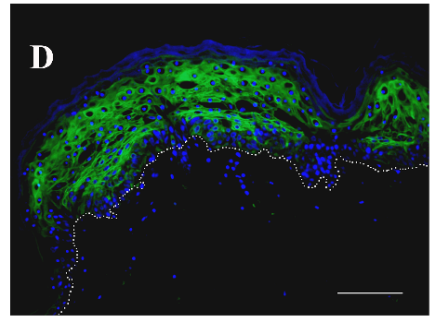

Figure 2. K17 immunostaining on paraffin skin sections. A: Normal skin; B-D: Samples incubated with medium containing TNF-alpha, IL-17, IL-22, and IL-23 at T24, T48, and T72. K17: Keratin 17. T24: Samples harvested 24 hours after the overnight incubation; T48: Samples harvested 48 hours after the overnight incubation; T72: Samples harvested 72 hours after the overnight incubation. Dotted white line indicates the basal membrane. Bars: $50 \mu \mathrm{m}$

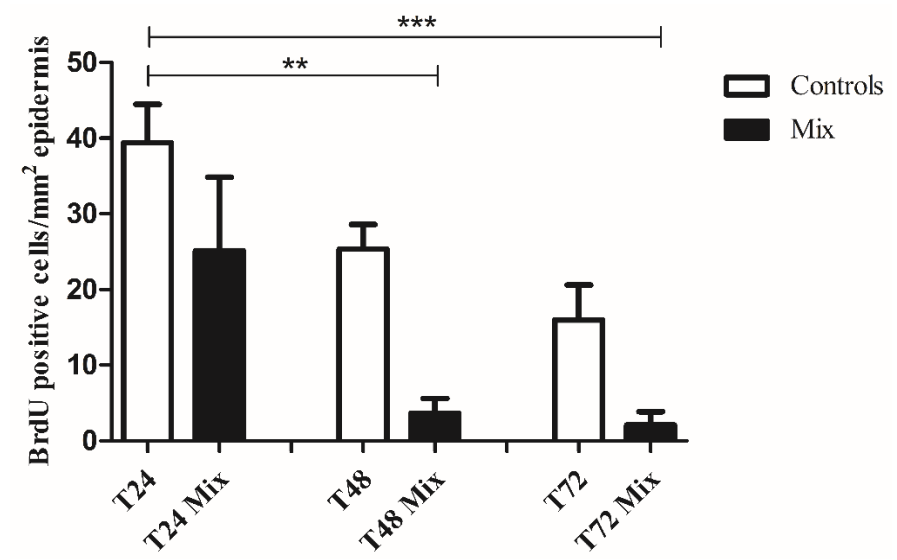

Figure 3. Keratinocyte epidermal proliferation. Keratinocyte epidermal proliferation in skin incubated with medium containing TNF-alpha, IL-17, IL-22, and IL-23 (Mix). T24: Samples harvested 24 hours after the overnight incubation; T48: Samples harvested 48 hours after the overnight incubation; T72: Samples harvested 72 hours after the overnight incubation

\section{Discussion}

The molecular mechanisms underlying the pathogenesis of psoriasis are well defined, but more specifically in the late phase of the disease. Much information concerning the very early pathogenic phases of this disease still lack. To investigate the onset of psoriasis, 3D models represent an useful approach and occupy an intermediate position between cell cultures and animal-based models, thus overcoming the multiple limitations existing in each proposed in vivo model [12]. The main advantage of the 3D model standardized in our lab is to reproduce the physiological condition since the skin is cultured in a Transwell system at the air-liquid interface with the epidermis exposed to the air and the dermis immersed in the culture medium. As no growth factors are normally added to the medium, it is possible to investigate the direct and prompt epidermal response to a psoriatic microenvironment [8-11].

In this study, we demonstrated that samples exposed to the cytokine mix share some pivotal morphological features of psoriatic skin mostly regarding K17 and TLR expression. The similarity is strictly dependent on the exposure time to the psoriatic microenvironment, strongly suggesting that the different psoriatic events are correlated to the duration of the specific interaction between epidermal cells and the exogenous proinflammatory psoriatic cytokines. Two main observations are noteworthy.

The first one is that the cytokine mix is able to induce the expression of TLR7 within all epidermal layers, highlighting, once again, the quick psoriatic change induced in the epidermal compartment if compared to healthy skin, where TLR7 immunoreactivity is never evident in the basal layer and is present only in the suprabasal compartment. Unpublished experiments obtained in our lab performed in the same experimental setting refer that a single cytokine does not succeed in inducing the expression of TLR7 at any time point. Thus, in pressed pro-inflammatory conditions mimicking the psoriatic environment, skin keratinocytes possibly prepare a strong response against immunopathogens primarily to avoid epidermal inflammations. Similar observations are reported in the literature; self-RNA-LL-37 is able to activate plasmacytoid dendritic cells via TLR7, which produce large amount of IFN-alpha potentiating $\mathrm{mDC}$ activity [14,15]. The activation of the TLR-7/nuclear factor kappa B (NF-kB) pathway has been shown to increase the production of several pro-inflammatory mediators that amplify the inflammation [16]. A synthetic TLR-7 agonist, Imiquimod (IMQ), was recently used in the clinic and it is reported to I) induce psoriasis and to II) exacerbate the disease in patients with well-controlled psoriasis. In experimental studies topical application of IMQ has been shown to induce psoriasislike skin inflammation through TLR-7 activation, along erythema and inflammatory cell infiltration in skin $[17,18]$.

According to the literature [19], TLR9 was never expressed in control samples supporting the hypothesis that, in absence of a proinflammatory stimulus, epidermis is actually quiescent. TLR9 expression was evident in the granular layer of mix samples in scattered granular keratinocytes starting from T24. Literature reported an overexpression of TLR9 in psoriatic lesional skin, but the Authors did not specifically describe the epidermal layer as they were more interested in showing the presence of the TLR9 than to the correlation between the receptor expression and keratinocyte stratification [19].

The second relevant finding regards the early induction of the expression of K17, an important and specific psoriatic marker. 
Interestingly, at the longest experimental time point, i.e. T72, K17 pattern distribution in the epidermal compartment of cytokine mix samples is very similar to the features if the psoriatic skin described in literature $[20,21]$. This observation indicates that normal epidermis is ready for early responding to a psoriatic microenvironment with a consequent prompt psoriatic shift, but the stimulus must persist over time, thus reproducing as strictly as possible the pathological condition in which pro-inflammatory psoriatic cytokines are involved.

The results obtained after the quantitative analysis of cell proliferation need a more complex explication because, as we all know, psoriasis is typically characterized by hyperproliferation, whereas we report in the present study an inhibition of keratinocyte proliferation after the exposure to the cytokine mix. As previously shown, in our experimental conditions, the reduction of cell proliferation can be interpreted as the prompt epidermal reaction leading later to the reparative proliferative response to injury found in psoriatic plaques [8]. In our studies, each single cytokine composing the mix, i.e. interleukin (IL-) 17, 22, 23 and tumour necrosis (TNF-) alpha, promptly induced a "psoriatic switch" with a high specificity [8-11]. In particular, each one exerted an intrinsic and specific effect on the expression of a wellknown psoriatic marker as keratin 17 [20].

\section{Conclusion}

In conclusion, our results prove that a psoriatic microenvironment is able to induce pivotal morphological changes in the epidermal compartment, modifying in particular the expression of TLR7, TLR9 and $\mathrm{K} 17$ in our $3 \mathrm{D}$ model. It gives also more details concerning the very specific localisation of these two receptors and this could be an important detail for the many new small molecules targeted against TLRs for the therapy of chronic inflammatory skin disease, above all psoriasis.

\section{Conflicts of interest}

The authors declare no potential conflict of interests.

\section{References}

1. Niehues H, van den Bogaard EH (2018) Past, present and future of in vitro 3D reconstructed inflammatory skin models to study psoriasis. Exp Dermatol 27: 512-519.

2. Lowes MA, Suárez-Fariñas M, Krueger JG (2014) Immunology of psoriasis. Annu Rev Immunol 32: 227-255. [Crossref]

3. Takahashi T, Gallo RL (2017) The Critical and Multifunctional Roles of Antimicrobial Peptides in Dermatology. Dermatol Clin 35: 39-50.
4. Fuentes-Duculan J, Bonifacio KM (2017) Autoantigens ADAMTSL5 and LL37 are significantly upregulated in active psoriasis and localized with keratinocytes, dendritic cells and other leukocytes. Exp Dermatol 26: 1075-1082.

5. Girolomoni G, Mrowietz U, Paul C (2012) Psoriasis: rationale for targeting interleukin-17. Br J Dermatol 167: 717-724. [Crossref]

6. Miller LS (2008) Toll-like receptors in skin. Adv Dermatol 24: 71-87. [Crossref]

7. Jeon YJ, Sah SK, Yang HS, Lee JH, Shin J, et al. (2017) Rhododendrin inhibits toll-like receptor-7-mediated psoriasis-like skin inflammation in mice. Exp Mol Med 49: e349. [Crossref]

8. Donetti E, Cornaghi L, Gualerzi A (2014) An innovative three-dimensional model of normal human skin to study the proinflammatory psoriatic effects of tumor necrosis factor-alpha and interleukin-17. Cytokine 68: 1-8.

9. Prignano F, Arnaboldi F, Cornaghi L (2015) Tumor necrosis factor-alpha and interleukin-17 differently affects Langerhans cell distribution and activation in an innovative three-dimensional model of normal human skin. Eur J Cell Biol 94: 71-77.

10. Donetti E, Cornaghi L, Arnaboldi F (2016) Interleukin 22 early affects keratinocyte differentiation, but not proliferation, in a three-dimensional model of normal human skin. Exp Cell Res 345: 247-254.

11. Donetti E, Cornaghi L, Arnaboldi F (2017) Epidermal barrier reaction to an in vitro psoriatic microenvironment. Exp Cell Res 360: 180-188.

12. Chiricozzi A, Romanelli M, Panduri S (2017) Relevance of in vitro 3-D skin models in dissecting cytokine contribution to psoriasis pathogenesis. Histol Histopathol 32 893-898.

13. Bedoni M, Sforza C, Dolci C (2007) Proliferation and differentiation biomarkers in normal human breast skin organotypic cultures. J Dermatol Sci 46: 139-142.

14. Diebold SS, Kaisho T, Hemmi H, Akira S, Reis e Sousa C (2004) Innate antiviral responses by means of TLR7-mediated recognition of single-stranded RNA. Science 303: 1529-1531. [Crossref]

15. Ganguly D, Chamilos G, Lande R (2009) Self-RNA-antimicrobial peptide complexes activate human dendritic cells through TLR7 and TLR8. J Exp Med 206: 1983-1994.

16. Miller LS (2008) Toll-like receptors in skin. Adv Dermatol 24: 71-87. [Crossref]

17. Stary G, Bangert C, Tauber M (2007) Tumoricidal activity of TLR7/8-activated inflammatory dendritic cells. J Exp Med 204: 1441-1451.

18. El Malki K, Karbach SH, Huppert J (2013) An alternative pathway of imiquimodinduced psoriasis-like skin inflammation in the absence of interleukin-17 receptor a signaling. J Invest Dermatol 133: 441-451.

19. Curry JL, Qin JZ, Bonish B, Carrick R, Bacon P, et al. (2003) Innate immune-related receptors in normal and psoriatic skin. Arch Pathol Lab Med 127: 178-186. [Crossref]

20. Jin L, Wang G (2014) Keratin 17: a critical player in the pathogenesis of psoriasis. Med Res Rev 34: 438-454. [Crossref]

21. Shen Z, Chen L, Liu YF (2006) Altered keratin 17 peptide ligands inhibit in vitro proliferation of keratinocytes and $\mathrm{T}$ cells isolated from patients with psoriasis. $J \mathrm{Am}$ Acad Dermatol 54: 992-1002.

Copyright: (C2019 Donetti E. This is an open-access article distributed under the terms of the Creative Commons Attribution License, which permits unrestricted use, distribution, and reproduction in any medium, provided the original author and source are credited. 